\title{
Trasplante hepático en niños
}

\author{
Erwin Buckel G. ${ }^{1}$; Sergio Ceresa O. ${ }^{1}$ : Mario Uribe M. ${ }^{1}$; José Zacarías S. ${ }^{1}$; \\ Carlos Carvajal H. ${ }^{3}$; Carlos Lizana S. ${ }^{\text {; }}$ Angel Blanco M. '; Jaime Cordero T. ${ }^{2}$; \\ René Tejías R. ${ }^{1}$; Emilio Santelices C. ${ }^{3}$; Alvaro Iñiguez V. ${ }^{3}$; Ramón Coloma O. ${ }^{3}$; \\ Jorge Morales B. '; Gladys Smok S. ${ }^{4}$; Cristina Herzog O. ${ }^{5}$
}

\section{Liver transplantation in children}

Irver ransplantation is the ireatment of chaice for a varie'y of chronic and acule terminal liver diseases. Until recently there was no et nical experience in liver transplantation in children in our country. This is a report cn 11 liver transplants done at Santiago. Chile, in children. Biliary alresia was the most frequent indication for the procedure. Among liver transplant recipients there were several high risk potients llow weight, multiple previous biliary surgical inlerventions, and a case of acule fulmınant hepatilis There were no intraperalive deaths. Donor liver reduction was necessary in four cases of small recipienls. Five potien!s (45\%) required treatmenl ogoinsl grafl rejection but there have been no groft losses due to this cause. Two recipients died early after transplantation, and ariother one, a patient wilh hepotoblostoma, died 15 months later. Actuarial one yeor patienl survival is $82 \%$. This is the first report on pediatric liver transplantation in this counlry, and our results in teras of survival and quality of lite prove that this procedure can be accomplished with similar results than in other places of Ihe world.

(Key words: liver Iransplantation, biliary arresic, chronic liver disease, hepatoblastoma.I

El trasplante hepático en niños se ha convertido en la opción terapéutica preferida para varias enfermedades hepáticas crónicas y agudas en fase terminal. En la última década ha habido progresos notables en la técnica quirúrgica, inmunosupresión y preservación de organos, aumentando la cantidad de trasplantes de hígado en todo el mundo, así como la sobrevida y la calidad de vida de los pacientes $^{1-3}$. La incorporación de este complejo procedimiento ha sido lenta en Chile, donde hasta 1993 no existía un programa activo de trasplante hepático pediátrico, lo que impedía emplearlo en un número significativo de niños que cumplian los requisitos para recibirlo. A fines de 1993 se inició la experiencia en este procedimiento en la Clínica Las Condes, de

1. Unidad de Trasplante de Organos. Clinica Las Condes.

2. Unidad de Cuidados Intensivos Pediátricos. Clínica Las Condes.

3. Departamento de Anestesiología. Clínica Las Condes

4. Departamento de Anatomía Patológica, U. de Chilc. Hospital J.J. Aguire.

5. Enfermera Universitaria. Coordinadora Programa de Trasplante de Organos, Climica Las Condes
Santiago Metropolitano. Desde entonces, el trasplante hepático ha sido hecho en niños y adultos con $80 \%$ de sobrevida a un año ${ }^{4}$. En esta primera comunicación sobre experiencias nacionales en trasplante hepático pediátrico se describen las características de los receptores, la selección de los pacientes, las indicaciones y complicaciones del procedimiento y la sobrevida de los beneficiados.

\section{Pacientes y Métodos}

Selección de receptores. Se consideraton candidatos a trasplante de higado todos los nifios referidos por diversas instituciones para tal proposito, en que el análisis de su caso por el equipo de trasplantes concluyó que no habian contraindicaciones y su enfermedad hepática era terminal. Especial enfasis se puso en la evaluación cardiovascular por la asociación de cardiopatías congénitas con atresia biliar. En aquellos niños con desnutrición severa se indicó asistencia nutricional intensiva, preferentemedte por sonda nasoyeyunal. La evaluación infeclologica se orientó especialmente a la identifiçación de virus de hepatitis $B$ y $C$, virus de inmunodeficiencia humana (VIH) y de reacciones séricas a organismos oportunistas que pueden complicar la evolución de? tras- 
plante (citomegalovirus, Candida albicans). Una vez completada la evaluación previa al trasplante, los candjdatos fueron incorporados a una lista de espera de acuerdo a la urgencia de su situación y grupo sanguíneo.

Procuración y distribución de órganos. Para obtener los órganos se utilizó lécnica de procuración multiorgánica, con canulación aórtica y portal y perfusión "in situ" con solución de preservación de Wisconsin ${ }^{5-7}$. La distribución de órganos se realizó de acuerdo a la compatibilidad de grupos sanguíneos y el tiempo de permanencia en la lista de espera del listado nacional en el Instituto de Salud Publica de Chile.

Método quírúrgico. Se utilizaron dos técnicas de inplantación en el receptor. En algunos casos en que los receplores pesaban mús de $20 \mathrm{~kg}$, se emplés el método de extirpación del hígado nativo con bomba de cortocircuito venoso-venoso ${ }^{k .9}$. En los casos restantes se usó la técrica de hepatechomía con vena cava "in-situ"li", que permite prescindir de la bomba y de la oclusión de vena cava du. rante el procedimiento. En otros casos fue necesario reducción el injerto por disparidad de volumen entre donante $y$ receptor, implantándose el lóbulo hepático izquierdo. La conexión biliodigestiva se realizó mediante anastomosis hepaticoyeyunal en Y de Roux en todos los casos.

Inmunosupresión y manejo de rcchazo. Sè usó un esquema inmunosupresor triasociado consistente en ciclosporina A, azatioprina y esteroides (inicialmente metilpredrisolona iv. seguido de prednisona oral), de acuerdo a un protocolo publicado anteriormente ${ }^{4}$. La vigilancia del rechazo se realizó en biopsias hepáticas percutóneas realizadas regularmente los días 7 y 21,5 meses y un año después del trasplante. De acuerdo a criterios histológicos establecidos ${ }^{11.12}$, Jos rechazos se clasificaron como leves, moderados o severos. En caso de signos histologicos de rechazo celular agudo, se adminisIraron al paciente bolos de netiiprednisolona de 20 $\mathrm{mg} \cdot \mathbf{k g} \cdot d o s i s$, día por medio por tres veces, al cabo de los cuales se repitió la biopsia hepática: si persistian los signos de rechazo se administraron anticuerpos policlonales (timoglobulina) por 10 a 14 días.

Análisis de resultados. Para el análisis de sobrevida de receptores se utilizó la estimación de sobrevida actuarial mediante el método de Kaplan Meier.

\section{Resultados}

Características de los receptores. Entre diciembre de 1993 y marzo de 1996 se realizaron 23 trasplantes hepáticos, 11 en niños. La edad, el sexo, peso y diagnóstico de estos últimos se describen en la tabla, donde destaca que siete eran mujeres, el promedio de la edad era 6,1 años, el peso fluctuaba entre $7 \mathrm{~kg}$ y 50 kg y la afección que con más frecuencia motivó el trasplante atresia biliar $(63,3 \%)$. Dos njños recibieron trasplante por insuficiencia hepática aguda (un caso de hepatitis fulminante y uno de hepatitis subfulminante). En un solo caso la indicacion del trasplante fue una neo- plasia maligna (hepatoblastoma difuso). En esta serie no se regisiran retrasplantes.

Antecedentes quirúrgicos. Todos los donantes y los receptores fueron intervenidos por el mismo equipo quirúrgico. El promedio de tiempo operatorio fue de 7,1 h (márgenes 4,5 a 11,0 h). El promedio de glóbulos rojos trasfundidos en el intraoperatorio fue de 0,41 $\mathrm{U} \cdot \mathrm{kg}$ (márgenes I a $30 \mathrm{U}$ por receptor). No se registraton muertes intraoperatorias. En cuatro casos $(36,3 \%)$ fue preciso reducir el injerto por desproporción entre el peso del donante y del receptor. En todos estos casos se usó el Ióbulo hepático izquierdo para hacer el implante. En todos los pacientes con atresia biliar y el receptor portador de síndrome de Alagille se había realizado algún procedimiento quirúrgico previo sobre la vía biliar (promedio 2,25 intervenciones previas por receptor, margenes I a 7). En tres receptores se requirió revascularizar la artcria hepática del injerto mediante un puente arterial desde la aorta (dos intrarrenales y uno supracelíaco). En todos los casos se reconstituyb la continuidad biliodigestiva mediante anastomosis hepaticoyeyunal en $Y$ de Roux, que en los casos de atresia bjliar se realizó con el asa de yeyuno del procedimiento de Kasai previo. En todos los casos se dejố un catéter biliar de acceso para colangiografías después del trasplante.

Evolución postoperatoria. La reintervención quirúrgica fue necesaria en cuatro receptores $(36.3 \%)$, debido a complicaciones en el postoperatorio. Una niña se reoperó por sepsis abdominal a Candida albicans, en otra se reali$z 6$ aseo peritoneal por hemoperitoneo secundario a coagulopatía postrasplante, en una tercera fue preciso reparar la arteria hepática por trombosis de la misma y en la cuarta reparar la vena porta, también por trombosis. Dos pacientes fallecieron precozmente despuśs del trasplante. Uno era un niño de 13 años sometido al procedimiento por hepatitis fulminante, con probable hepatotoxicidad por färmaco $e$ insuficiencia hepática grave, en quien el transductor intraventricular registraba cifras aceptables de presión intracraneana antes y durante el trasplante, sin embargo, en el postoperatorio -a pesar que el injerto funcionaba bien- desarrollo hipertensión endocraneana y murió a causa de ella. Otra paciente murió en insuficiencia hepática debido a trombosis precoz de vena porta, a pesar de 
Tabla

Características demográficas y enfermedad que causó la indicación de trasplante hepático en once pacientes menores de 15 años

\begin{tabular}{|c|c|c|c|c|c|}
\hline $\begin{array}{c}\text { Paciente } \\
\text { (n) }\end{array}$ & Fecba TH & $\begin{array}{l}\text { Edad } \\
\text { (años) }\end{array}$ & Sexo & $\begin{array}{l}\text { Peso } \\
(\mathrm{kg})\end{array}$ & Diagnóstico \\
\hline 1 & $14-12-93$ & 2,0 & $\mathrm{~F}$ & 12 & Hepatoblastoma \\
\hline 2 & $03-03-94$ & 12,0 & $\mathrm{~F}$ & 50 & Atresia vfa biliar \\
\hline 3 & $16-03-94$ & 11,0 & $\mathrm{~F}$ & 29 & Subfulminante \\
\hline 4 & $03-08-94$ & 6.0 & $\mathrm{~F}$ & 25 & Atresia yía biliar \\
\hline 5 & $08-10-94$ & 1,9 & $\mathrm{M}$ & 7 & S. de Alagille \\
\hline 6 & $10-03-95$ & 7,0 & $\mathrm{~F}$ & 24 & Atresia vía biliar \\
\hline 7 & $05-07-95$ & 8.0 & $M$ & 26 & Atresia vfa biliar \\
\hline 8 & $07-11-95$ & 2,0 & $M$ & 10 & Atresia vía biliar \\
\hline 9 & $20-12-95$ & 13,0 & $M$ & 44 & Fuldminante vía biliat \\
\hline 10 & $11-03.96$ & 4,0 & $\mathrm{~F}$ & 18 & Atresia vía biliar \\
\hline 11 & $19-03.96$ & 0.8 & $F$ & 7 & Atresia wa biliar \\
\hline
\end{tabular}

rH: trasplante hepatico

haber sido reoperada. El promedio de días de hospitalización en cuidados intensivos de los sobrevivientes fue 6,5 días (márgenes 2 a 27 dias) y el promedio de las duraciones de la hospitalización completa para trasplante fue 25,6 días (márgenes 9 a 56 días). No se registraron complicaciones que afectasen la vía biliar en el postoperatorio precaz.

Complicaciones inmunológicas. Seis receptores $(55 \%$, casos $1,3,4,6,8,10)$ presentaron durante su evolución un episodio de rechazo celular agudo (RCA), confirmado histológicamente. Todos los pacientes, salvo el caso 1 (leve, sin manifestaciones bioquímicas de rechazo y cursando con una infección severa) recibieron metilprednisolona iv en bolos y una (caso 4, moderado, resistente a esteroides) requirió, además, tratamiento con anticuerpos policlorales durante 10 días (Timoglobulina ${ }^{\mathrm{R}}$, Rhodia Merieux). Al momento de esta publicación no se habían perdido injertos o pacientes por rechazo y ninguno de los receptores estaba en riesgo de que ello ocurriese.

Complicaciones infecciosas. Dos pacientes tuvieron infecciones por gérmenes oportunistas. Uno sufrió una sepsis con foco abdominal por Candida albicans que se resolvio con cirugía abdominal y administración prolongada de anfotericina B. Otro presentó una neumonitis por citomegalovirus, confirmada por cultivo del agente en lavado broncoalyeolar, la que fue tratada con ganciclovir durante 14 días. Otros dos receptores tuvieron bacteremia a diversos gérmenes durante el período postoperatorio precoz, debidas a contaminación de accesos vasculares, las que fueron controladas con antibióticos de uso frecuente.

Otros tipos de morbilidad posterior al trasplante. Una niña curso con insuficiencia renal aguda después del trasplante, que obedeció a varias causas y requirió transitoriamente apoyo con hemodiálisis, siendo normal su funcion renal posteriormente. En otro paciente se registraron convulsiones como signo de neurotoxicidad por ciclosporina, que respondieron favorablemente a tratamiento con anticonvulsivantes y ajuste de las concentraciones séricas de ciclosporina.

Complicaciones alejadas. La complicación tardía más grave de la serie ocurrío en el niño sometido a trasplante por hepatoblastoma, en quien la lesión reapareció en el pulmón $8 \mathrm{me}$ ses después del trasplante, siendo tratado entonces con resección quirúrgica y quimioterapia, no obstante lo cual el tumor volvió a aparecer, esta vez en el mediastino, causándole la muerte. No se encontró recurrencia de la enfermedad en el hígado trasplantado y el abdomen. En dos receptores se han registrado complicaciones biliares tardías: uno muestra múltiples estenosis biliares intrahepáticas sin causa aparente, ha evolucionado con episodios 
recurrentes de colangitis y probablemente su solución definitiva sea un nuevo trasplante de hígado; el otro caso tuvo trombosis de arteria hepática que fue reparada, evolucionó con necrosis aséptica del lóbulo hepático izquierdo que se manejó en forma conservadora, posteriormente se desarrolló una estenosis del conducto hepático común, proximal a la anastomosis biliodigestiva, la que ha sido manejada con un catéter biliar transhepático percutáneo y dilataciones progresivas, con buen resultado. Otro receptor sufrió tardíamente aplasia de la médula ósea con pancitopenia, causada probablemente por medicamentos, que respondió satisfactoriamente al modificar el tratamiento y administrar productos sanguineos.

Sobrevida. Tres de 11 niños que han recibido trasplantes hepáticos han fallecido: dos en el período postoperatorio (uno por hipertensión endocraneana postoperatoria con injerto funcionante, otro por trombosis de vena porta e insuficiencia hepática respectivamente); uno 15 meses después del trasplante por recidivas extraabdominales del hepatoblastoma inicial. Actualmente sobreviven 9 pacientes $(73 \%$ ), con seguimientos de 3 a 27 (promedio 16) meses y $82 \%$ sobrevida actuarial a 12 meses, quienes cstán en excelentes condiciones (cinco mayores estudian y tres menores están en actividad), salvo el paciente 5 con sindrome de Alagille, que sufre colangitis recurrente y en quien se ha planteado la posibilidad de un nuevo trasplante.

\section{Comentario}

Los trasplantes hepáticos son una buena opción terapéutica en niños y adultos. Ofrecen a pacientes con insuficiencia hepática terminal, probabilidades de sobrevida de buena calidad de alrededor de $70 \%$ a largo plazo ${ }^{13-16}$. La serie que se comenta, aun con pequcño número de casos, es una muestra inicial de los beneficios de un programa en expansión. En ella también la indicación más frecuente fue la atresia biliar. Los beneficios del trasplante hepático en niños con colestasia severa e insuficiencia hepática secundaria a atresia biliar han sido ampliamente demostrados ${ }^{17-19}$, aunque estos pacientes suelen presentar dificultades técnicas adicionales al trasplante, por sufrir hipertensión portal y haber sido, frecuentemente, sujetos de uno o más procedimientos previos de cirugía biliar. La indicación de trasplante hepático en pacientes con tumores malignos del hígado es motivo de grandes controversias. Se acepta la indicación en ciertos casos muy bien seleccionados de hepatoblastomas, a pesar que los resultados son inferiores a los que se obtienen en afecciones no neoplásicas ${ }^{20,21}$. En el único caso en esta situación de esta serie no se logró controlar efectivamente el tumor ${ }^{22-24}$.

Esta serie incluye un número considerable de pacientes considerados habitualmente en alto riesgo, por factores como desnutricion, cirugías abdominales múltiples, hipertensión portal severa y peso corporal inferior a $10 \mathrm{~kg}$ y se debe a que la intención del programa fue resolver un problema a niños en que había in. dicación perentoria de trasplante, y no la de seleccionar casos menos complejos. Para ha cer el trasplante en estos niños se emplearon técnicas quirúrgicas de vanguardia, adecuadas selectivamente a las necesidades particulares de cada receptor, como su peso y magnitud de la hipertensión portal. Algunas, como las reducciones hepáticas y la preservación de la vena cava no se habían empleado antes en Chile y, por lo tanto, su introducción es mérito de cste programa. La incidencia de complicaciones vasculares (trombosis de arteria hepática, trombosis de vena porta) es la esperada en cualquier serie de trasplantes hepáticos ${ }^{25.26}$ y guarda relación con las dificultades técnicas señaladas anteriormente. La morbilidad por infecciones estuvo representada fundamentalmente por patógenos oportunistas frecuentes en pacientes inmunosuprimidos ${ }^{27,28}$. Nuestro protocolo de profilaxis de infecciones incluye el empleo rutinario postrasplante de aciclovir oral en dosis altas $\left(2 \mathrm{~g} \cdot \mathrm{m}^{2}\right)$ y, en casos de alto riesgo (reccptor IgG negativo o donante IgG positivo para cilomegalovirus) ganciclovir iv durante 14 días y a continuación aciclovir oral. El control de las reacciones de inmunidad es probablemente donde más se ha progresado en el área de trasplantes, con esquemas cada vez más específicos, efectivos y menos lóxicos, pero también más caros. El empleado en esta experiencia, que incluye ciclosporina A como el inmunosupresor fundamental, es de probada efectividad y el más utilizado en el mundo ${ }^{29-32}$. Es muy poco probable que la baja 
incidencia de rechazo registrada sea subestimación de la real, ya que nuestro protocolo de seguimien to ${ }^{4}$ incluye biopsias hepáticas de rutina, además de las que se hacen por necesidad cuando hay signos clínicos o bioquímicos de rechazo, y todos los casos fueron confirmados histológicamente. Estas cifras son muy favorables, pues la incidencia de rechazo fluctúa habitualmente entre $50 \%$ y $75 \% 29,30,33$. Nuestro actual protocolo comprende una nueva formulación de ciclosporina A (microemulsionada. Neoral $\left.{ }^{(R)}\right)^{34-36}$; y el cambio del antimetabolito azatioprina por micofenolato, mucho más efectivo, en la profilaxis y el tratamiento de recha$\mathrm{z}^{37}$. Creemos que con estos agentes podríamos mejorar aún más los resultados. La mortalidad en esta serie obedeció sólo en un caso a una complicación directamente relacionada con el procedimiento quirúrgico (trombosis de vena porta e insuficiencia del injerto). En hepatitis fulminante la indicación de trasplante y el momento de hacerlo son materia de controversias, pero los resultados suelen ser satisfactorios (aunque no tanto como en casos electivos), cxcepto en pacientes con infecciones activas, hipertensión endocraneana o falla multiorgáni$\mathrm{ca}^{38}$. Nuestro caso de este tipo ilustra el alto riesgo de la situación, la dificultad para lograr una apropiada selección de los pacientes y de poder someterlos al procedimiento, ante la irregular disponibilidad de donantes en nuestro medio. La sobrevida actuarial a 12 meses de nuestros pacientes es comparable a la obtenida en centros más grandes, experimentados y bien dotados de recursos ${ }^{39,40}$. La calidad de vida de los receptores es excelente en la mayoría de los beneficiarios del procedimiento, con reintegración a una vida prácticamente normal. Esta experiencia preliminar demuestra que en Chile, a pesar de las dificultades propias del medio, como bajo número de donantes, limitaciones económicas y referencia tardía de los potenciales candidatos, se pueden lograr resultados satisfactorios en trasplantes hepáticos.

\section{Resumen}

Los trasplantes hepáticos constituyen el tratamjento más adecuado en gran número de pacientes con cnfermedades crónicas y agudas terminales del hígado. La experiencia con este procedimiento es muy limitada en Chile. Se describen 11 niños que recibieron trasplantes hepáticos. La indicación mas frecuente para hacerlo fue la atresia hiliar. Se incluyeron receptores de alto riesgo como peso inferior a $10 \mathrm{~kg}$, numerosas intervenciones quirúrgicas previas $y$ hepatitis fulminante, pero no se registraron muertes intraopcratorias. En cuatro casos (36\%) fue necesario hacer reducciones del hígado de los dadores para acomodarlos a receptores de bajo peso. Cinco receptores (45\%) fueron tratados por rechazo agudo $y$ no se han perdido injertos por causa inmunológica. Dos receptores fallecieron precozmente (uno por trombosis porta, otro por hipertensión intracraneana) y uno 15 meses después del trasplante por recidiva pulmonar y mediastínica de hcpatoblastoma. La sobrevida actuarial es, hasta la fccha, $82 \%$ en un año. La serie demuestra que es posible realizar estos complejos procedimientos en el país, con similares resultados que en otros lugares del mundo.

(Palabras clave: trasplante hepático, atresia de vías biliares, enfermedades hepáticas crónicas, hepatoblastoma.)

\section{Agradecimientos}

A las unidades y personal de cuidados intensivos e infecciosos del Hospital Luis Calvo Mackenna por su colaboración en el cuidado de algunos de estos pacientes en sus dependencias.

\section{Referencias}

1. Belle SH. Beringer KC. Detre $K$, et ul: Trends in liver transplantation in the Uniled States. In: Terasaky PI, Cecka JM, Eds. Clinical Transplants 1993. Los Angeles. UCLA Tissue Typing Laboratory 1994; 19-35.

2. Worldwide Transplant Center Directory. In: Terasaky PJ, Cecka JM. Eds. Clinical Transplants 1993. Los Angeles, UCLA Tissue Typing Laboratory 1994 691-705.

3. Hepp J. Zaror M. Rios $H$., el al: Perspeclivas del Irasplante hepático en Chile. Rev Med Chile 1990 118: 453.458 .

4. Buckel E, Silva G, Brahm J, et aI: Experiencia de un ceniro en trasplante hepático en adultos y nifjos. Rev Med Chile 1996; 12: 27-36.

5. Starzl TE, Hakala $T$. Shaw B, et al: A flexible procedure for multiple cadaveric organ procurement. Surg Gynecol Obstet 1984: 158: 223-230.

6. D'Alessandro AH. Kalayogly M. Belzer FO: Experience with Belzer UW cold storage solution in 
human liver transplantation. Transpl Proc 1990 ; 22: 474-476.

7. Kalayogiu $M$. Belzer FO: Extended preservation of the liver for clinical transplantation. Lancet 1988; 19: 617-619.

8. Shaw B, Martin DJ. Marquez $J M$, et al: Venous bypass in clinical lives Iransplantation. Ann Surg 1984: 200: 524-534

9. Shaw B. Martin DJ. Márquez JM, et al: Advantages of venous bypass during orthotopic transplantalion of the liver. Semin Liver Dis $1985 ; 5: 344-348$.

10. Tzakis A. Todo \$, Starzl TE. et al: Orthotopic liver transplantation with preservation of the inferior vena cava. Ann Surg 1989: 210: 649-562.

11. Ladwig f: Classification and Ierminology of hepatic allograft rejection: whither bound? Mayo Clinic Proceedings 1989; 64: 676-679.

12. Ludwig $J$; Terminology of hepatic allograft rejection (glossaty). Seminars Liver Disease 1992; 12: 89-92.

13. Health Resources and Services Administration. Bureat uf Healih Resources Development: 1991 Report of center-specific graft and patient survival rates. Rockville. MD. US Department of Health and $\mathrm{Hu}$ man Services 1992: 8-10.

14. Belle SH, Detre KM: Report from the Pitt-Unos liver transplant registry. Transplant Proc 1993; 25: 1 137-1142.

15. Iwarsuki S. Starzl TE, Todo S. et al: Experience in 1000 liver transplants under cyclosporine and steroid therapy: a survival report. Transplant Proc 1988; 20: 488-504.

16. Busutil $R W$, Colonna JO, Hiatt $J R$, et al: The first I00 liver transplants at UCLA. Ann Surg 1987; 206: 387-402

17. Busutrit $R$ : Liver transplantation in children. Ann Surg 1991; $213: 48-57$

18. Hiatt JE. Ament ME. Berquist WJ, et al: Pediatric liver transplantation at UCLA. Transpl Proc 1987 . 19: 3282-3288.

19. Iwassuki S, Shaw f, Starzl TE: Liver transplantation for biliary atresia. World J Surg 1984: 8: 5]-56.

20. Koneru B, Flye W, Busuttil RW, et al: Liver transplantation for hepatoblastoma. The american experience. Ann Surg 1991; 213: 118-12k.

21. Penn I: Hepatic transplantation for primary and metastatic cancer of the liver. Surgery 1991; 110:726-735.

22. Paya CV, Hermans PE. Washington SA. et al: Incidence, distribution and outcome of episodes of infection in 100 orthotopic liver transplantalion. Mayo Clin Proc 1989; 64: 555-564.

23. Kusne S. Starzl TE. Ho M. et al: Infections in liver transplantation. Medicine 1988; 67: 132-143.
24. Ho $M$ : Advances in understanding cytomegalovirus infection after transplantation. Transplant Proc 1994: $26: 7-9$.

25. Tan KC, Yandza $T$, de Hemtinne B, et al; Hepatic artery thrombosis after pediatric liver transplantotion. J Pediatr Surg 1988: 23; 927-930.

26. Stieber AC, Zetti $G$, Todo $S$ : The spectrum of portal vein thrombosis in liver transplantation. Ann Surg 1991; 213; 199-206.

27. Pava CV. Hermans PE, Wiesner RH, ef al: Cytomegalovirus hepatitis in liver transplantion: analysis of 93 consecutive orthotopic liver transplantation J Infect Dis 1989; 160: 752-758.

28. King M. Petric $M$. Superina RA: Cytomegalovirus infections in pediatric liver transplantation. Am J Dis Child 1990; 144: 1307-1310.

29. Lake JR, Roberis JP, Ascher NL: Maidtenance immunosuppression after liver transplantation. Semin Liv Dis 1992; 12: 73-79.

30. Adams DH, Neuberger IM: Treatment of acute rejection. Semin Liv Dis 1992: 12: 80-88.

31. Pudbury RT, Gunsom BK, Dousset B, et at: Long term immunosuppression after liver transplantation. Transplant Int 1992; 5 ; $S 470-\$ 472$.

32. Starl TE, Xlintmalm GB, Porter KA, et al: Liver transplantation with use of cyclosporin $A$ and prednisone. N Engl J Med 1981; 305: 266-269.

33. Klintmain $G$, Nery $J$, Husberg $B$, ef at: Rejection in liver transplantation. Hepatology 1989; 6: 978-985.

34. Levy $G$. Alraif $M$, Reziev $S$, et al: Cyclosporine neoral in liver transplant recipients. Transpl Proc 1994; 26: $3184-3187$.

35. Farges $O$. Haddad N, Bismuth $H$. et al: Conversion from cyclosporine Sandinmun to cyclosporine Neoral in liver transplant patients. Transpl Proc 1995; 27: $2506-2,507$

36. Levy GA. Grent $D$ : Neoral in liver transplantation, Transpl Proc 1996; 28: 1019-1021.

37. Soltinger HW: Mycophenolate mofetil for the prevenlion of acute rejeclion in primary cadaveric renal allograft recipients. Transplantalion 1995: 60: 225-232.

38. Williums R. Wendon $J$ : Indications for orthotopic liver Iransplantation in fulminant liver failure. Hepatology 1994; 20: 5S-10S.

39. Ryckman F. Fisher $R$, Pedersen $S$. et al: Liver transplantation in children. Seminars in Pediatric Surgery 1992; 1: I62-172.

40. Bilik $R$, Greig $P$. Langer B, Superina $R$ : Survival after pediatric liver transplantation is dependent on pretransplant status. J Pediatr Surg 1993; 28; 1307-1311. 